\title{
Diagnostische Lumbalpunktion
}

Klaus Gröschel, Sonja Gröschel

Besteht der Verdacht auf eine entzündliche Erkrankungen des Nervensystems, ist eine Liquoranalyse indiziert. Gewonnen wird das „Nervenwasser“ meist durch eine Punktion des

Duralsacks im Bereich der Lendenwirbelsäule. Lesen Sie hier, wie so eine Lumbalpunktion abläuft.

Bei dem vorliegenden Artikel handelt es sich um eine überarbeitete Version von K. Gröschel, S. Schnaudigel, S. M. Pilgram, K. Wasser, A. Kastrup. The diagnostic lumbar puncture, Dtsch Med Wochenschr 2008; 133: 39-41

Indikationen | Eine diagnostische Lumbalpunktion ist indiziert bei:

- entzündlichen Erkrankungen (Meningitis, Multiple Sklerose, Neuroborreliose, Polyradikulitis)

- Nachweis einer Subarachnoidalblutung

- Liquordruckmessung

- neurodegenerativen Erkrankungen (z. B. Demenzen)

- Nachweis von Tumorzellen

- therapeutischen Maßnahmen: idiopathische intrakranielle Hypertension, Medikamenteninstillation, lumbale Drainage bei Liquorresorptionsstörung

Kontraindikationen | Eine Lumbalpunktion sollte nicht durchgeführt werden bei:

- Gerinnungsstörungen

- Thrombozyten $<50000 / \mu$ l als relative, $<20000 / \mu$ l als absolute Kontraindikation

- Quickwert< $<0 \%$, INR $>1,7$

- lokalen Entzündungen (Gefahr der Keimverschleppung)

- erhöhtem intrakraniellen Druck (Gefahr der zerebralen Einklemmung)

Abb. 1 Materialien für die Lumbalpunktion: (1) sterile Handschuhe, (2) Lochtuch, (3a) atraumatische Sprotte-Nadel mit Introducer (3b) QuinckeNadel mit angeschrägtem Schliff, (4) Liquorröhrchen, (5) Tupfer, (6) Monovette, (7) Utensilien für eine Lokalanästhesie

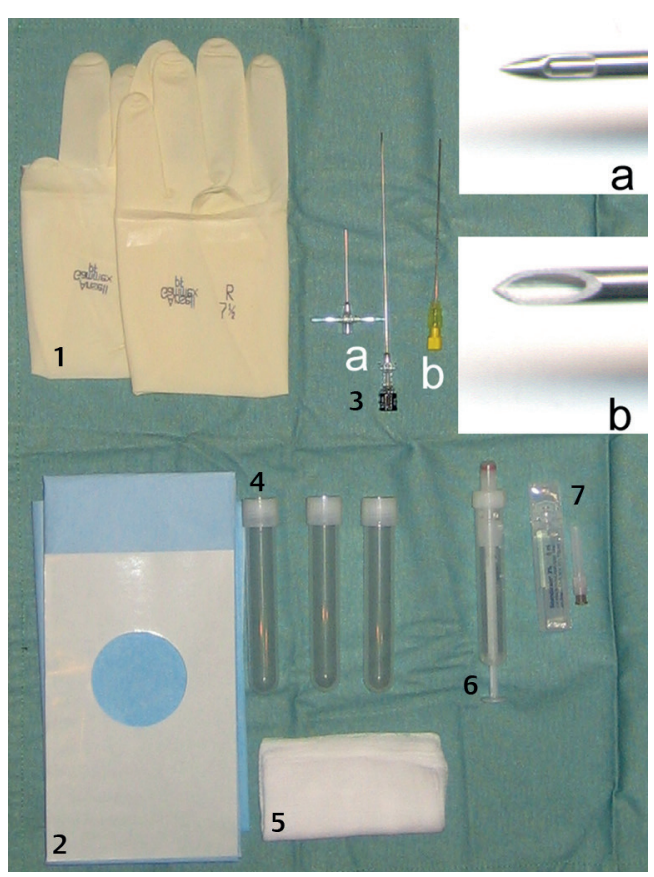

- kardiorespiratorischen Erkrankungen, die die notwendige Lagerung des Patienten nicht erlauben

*Acetylsalicylsäure 100 mg oder niedermolekulare Heparine in low-dose sind keine Kontraindikationen.

Patientenaufklärung I Nach einer Risiko-NutzenAbwägung und dem Ausschluss von Kontraindikationen wird der Patient über die Prozedur und die möglichen Nebenwirkungen ( $\bullet$ Tab. 1) aufgeklärt. Dabei helfen vorgefertigte Aufklärungsbögen.

Hirndruck überprüfen | Bei Patienten mit Vigilanzminderung oder neurologischen Auffälligkeiten muss man vor der Punktion einen erhöhten Hirndruck - und damit die drohende Gefahr einer zerebralen Einklemmung - ausschließen. Dies geschieht durch eine cerebrale Bildgebung (Computer- oder Magnetresonanztomografie). Die Spiegelung des Augenhintergrunds, zum Ausschluss einer Stauungspapille, genügt nicht. Die Prämedikation mit einem Benzodiazepin (z.B. Lorazepam) hat sich bei ängstlichen Patienten bewährt.

Antikoagulantien | Es gibt derzeit keine klaren Empfehlungen, wie lange direkte orale Antikoagulantien (Apixaban, Dabigatran, Rivaroxaban) vor dem Eingriff abgesetzt sein sollten.

Aus pathophysiologischen Überlegungen sollte der zeitliche Abstand von der letzten Einnahme bis zum Eingriff mindestens zwei Halbwertszeiten der jeweiligen Substanz betragen. Ein „Bridging“ mit Heparin ist nicht indiziert [4].

Material | Für die Lumbalpunktion benötigen Sie ( Abb. 1):

- sterile Handschuhe, Tupfer und Lochtuch

- Punktionsnadel

- atraumatische Sprotte-Nadel mit Introducer

- oder Quincke-Nadel mit angeschrägtem Schliff

- Liquorröhrchen

- Utensilien für eine Lokalanästhesie

- Monovetten für Blutabnahme

- Steigrohr zur Liquordruckmessung

- Mundschutz 


\section{So wird's gemacht: Lumbalpunktion}

Patientenlagerung | Am einfachsten lässt sich der Patient im Sitzen („Katzenbuckel“) z.B. auf der Bettkante punktieren. Stabilisiert wird er durch ein großes Kissen im Schoß und einem Stuhl zum Abstützen der Beine. Die Wirbelsäule sollte lotrecht, die Schulter- und Beckenlinie parallel zueinander stehen ( $\triangleright$ Abb. 2a). Ist eine Liquordruckmessung geplant, oder der Patient immobil bzw. unkooperativ, sollte die Punktion in Seitlagerung („Embryohaltung“, > Abb. 2b) erfolgen. Eine Liquordruckmessung wird vor Abnahme der Liquorprobe am liegenden Patienten vorgenommen.

Punktionsstelle finden | Erfühlen Sie mit den Händen die Beckenkämme des Patienten - auf einer gedachten Verbindungslinie lässt sich so mit den Daumen der Dornfortsatz des 4. Lendenwirbelkörpers (LWK) ertasten. Als mögliche Punktionshöhe bieten sich die Zwischenräume LWK 3 / 4, LWK 4 / 5 oder LWK 5/S 1 an - jeweils $1 \mathrm{~cm}$ unter dem zu tastenden Dornfortsatz von LWK 3, 4 oder 5.

Markieren Sie die zu punktierende Stelle durch einen leichten Druck mit dem Fingernagel oder einem Hautstift - so finden Sie die Stelle nach Desinfektion und Abdeckung leichter wieder.

Desinfektion und Anästhesie | Das entsprechende Hautareal wird desinfiziert und nach Abtrocknung ein steriles Lochtuch aufgelegt. Ob eine Gesichtsmaske getragen werden sollte, wird kontrovers diskutiert. Obligatorisch ist sie bei:

- immungeschwächten Patienten

- respiratorischen Infekten des Untersuchers

- vermeintlich langdauernder Punktion

Sie sollten dem Patienten eine Lokalanästhesie anbieten. Hierbei ist auf Allergien und eine erhöhte Infektionsgefahr zu achten.

Verschiedene Nadeltypen | Für die Punktion stehen zwei Nadeltypen zur Auswahl:

1. die „atraumatische“ Sprotte-Nadel (meist mit einem separaten Introducer) $(\triangleright$ Abb. $1 \mathbf{a})$

2. die ,traumatische“ Quincke-Nadel $(\triangleright$ Abb. 1b) Die Sprotte-Nadel senkt die Inzidenz postpunktioneller Kopfschmerzen. Die Quincke-Nadel ist vor allem bei eiweiß- oder zellreichem Liquor geeignet, da sie einen dickeren Innendurchmesser und dadurch einen geringeren Flusswiderstand hat.

Mit steigender Nadelgröße nehmen postpunktionelle Beschwerden (insb. Kopfschmerzen) deutlich zu. In der Routinediagnostik ist daher die schmalere Sprotte-Nadel vorzuziehen.

Hautdurchstich und Einführen der Nadel | Nach steriler Anreichung wird mit dem Introducer (scharf geschliffene Spitze, Schliffrichtung ent-

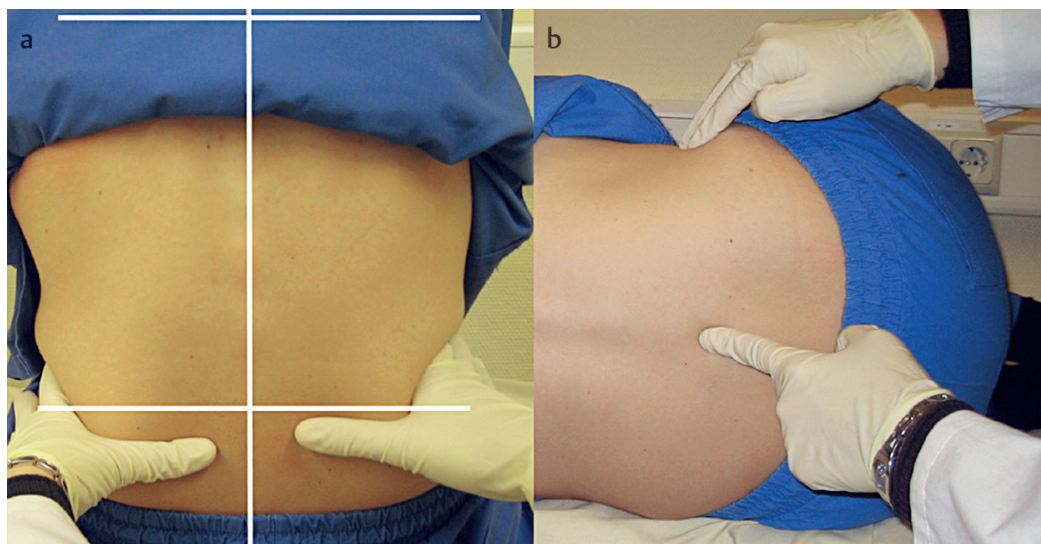

Abb. 2 Lagerung des Patienten. (a) Im Sitzen (b) Embryohaltung.

lang den Spaltlinien der Haut richten) die Haut durchstochen. Die Sprotte-Nadel (stumpfe Spitze) führt man anschließend durch den Introducer hindurch $(\checkmark$ Abb. 3). Die Nadel wird zwischen den Wirbelfortsätzen in Richtung Spinalkanal vorgeschoben. Dabei ist die Nadelführung ca. $20^{\circ}$ nach kranial und streng sagittal in Richtung Bauchnabel gerichtet ( Abb. 4 ).

Durchstechen der Dura | Das Ligamentum flavum macht sich in circa $3 \mathrm{~cm}$ Tiefe als Widerstand bemerkbar. Dieser gibt spürbar nach, sobald die Dura durchstochen ist. Die Nadelspitze gelangt in den Liquorraum. Bei Verwendung einer traumatischen Nadel sollte deren Öffnung vor dem Duradurchstich parallel zur Faserrichtung liegen (also senkrecht). Hierdurch werden die Fasern eher beiseitegeschoben als durchtrennt. Wenn Sie den Mandrin zurückziehen, können sie testen, ob sich die Kammer am Nadelende mit Liquor füllt und die Punktion erfolgreich war ( Abb. 5).

Nadelposition evtl. korrigieren | Sollte kein Liquor sichtbar sein, hilft es oft, die Nadel zu drehen oder leicht zu versetzen. Für letzteres schiebt man den Mandrin erst wieder ein, zieht ihn dann zusammen mit der Nadel bis zur Unterhaut zurück (ohne die Haut zu verlassen) - und schiebt ihn wieder in einem leicht veränderten Winkel ein. Berichtet der Patient von radikulären Missempfindungen, die in die Beine ausstrahlen, wurde die Nervenwurzel touchiert. Die Nadelposition ist dann meist zu weit lateral.

Sammeln des Liquors | Nach erfolgreicher Punktion lässt man die benötigte Liquormenge $(10-15 \mathrm{ml})$

Tab. 1 Häufige und seltene Nebenwirkungen einer Lumbalpunktion. durch den Introducer in Richtung Spinalkanal vorgeschoben.

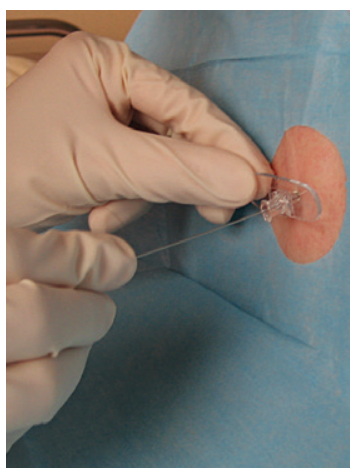

Abb. 3 Die Sprotte-Nadel wird

\section{häufig (>3\%)}

- lokaler Schmerz, ggf. mit vegetativen Kreislaufreaktionen

- postpunktionelle Kopfschmerzen, Übelkeit, Rückenschmerzen

- kurz ausstrahlende Missempfindungen in die Beine bei Berührung der Nervenwurzel

\section{selten}

- Verletzung von Blutgefäßen mit Subdural- oder Epiduralblutung

- Auftreten lokaler Entzündungen, ggf. auch Meningitis

- passagere Hirnnervenausfälle

- zerebrale Einklemmung

- Sinus- und Venenthrombose 
Abb. 4 Sagittales T2w-MRT der Wirbelsäule. Der Conus medullaris endet in $94 \%$ zwischen LWK 1/2. Die Spitze der Nadel (siehe Strich) liegt im Liquorraum zwischen LWK 3/4. Der Liquor ist in dieser T2-gewichteten Aufnahme hell dargestellt. Die grauen Stränge innerhalb des Liquorraumes entsprechen der Cauda equina des Rückenmarkes (Abdruck mit freundlicher Genehmigung des Instituts für Neuroradiologie, Universitätsmedizin Mainz)

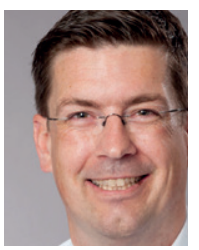

PD Dr. med. Klaus Gröschel ist Oberarzt an der Klinik und Poliklinik für Neurologie an der Universitätsmedizin Mainz. klaus.groeschel@unimedizinmainz.de

Dr. med. Sonja Gröschel ist Assistenzärztin an der Klinik für Psychiatrie und Psychotherapie an der Universitätsmedizin Mainz

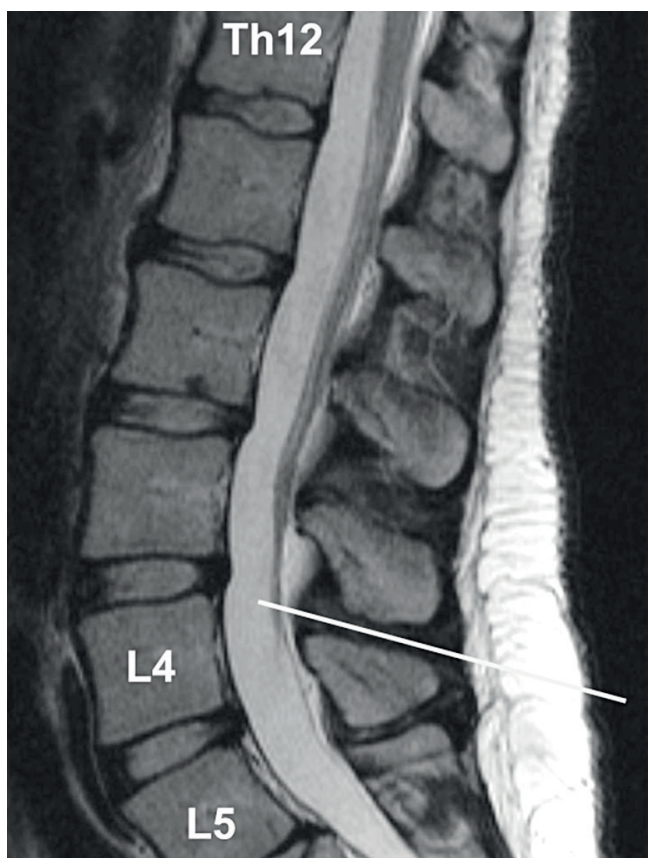

nacheinander in drei Röhrchen tropfen (3-GläserProbe). Artifiziell blutig tingierten Liquor erkennt man daran, dass das letzte Röhrchen klarer als die vorangehenden ist ( $\mathbf{A b b}$. 6 a). Pathologische Blutbeimengungen oder xantochromer Liquor ( $\triangleright$ Abb. 6b) ändern ihre Farbe nicht.

Entfernen der Nadel | Vor dem endgültigen Entfernen der Nadel muss der Mandrin wieder eingeschoben werden - hierdurch lassen sich postpunktionelle Kopfschmerzen reduzieren. Nach Abnahme werden Liquor - und meist auch eine aktuelle Blutprobe - zeitnah analysiert.

\section{Nach dem Eingriff}

Therapie bei postpunktionellen Kopfschmerzen I Die häufig auftretenden postpunktionellen Kopfschmerzen werden wahrscheinlich durch ein Nachsickern von Liquor durch das iatrogene Duraleck verursacht. Sie sind meist bifrontal oder -occipital betont und von dumpf-ziehendem Charakter. Eine prompte Besserung tritt auf, wenn sich der Patient hinlegt. Zusätzlich helfen NSAIDs, oder - bei Persistenz - Theophyllin $(1050 \mathrm{mg} / \mathrm{d})$ oder Koffein (600-1200 mg/d). Kommt es zu kei-

Abb. 6 (a),3-Gläser-Probe“ bei artifiziell blutig tingiertem Liquor. (b) Xanthochromer Liquor, z. B. nach subarachnoidaler Blutung.

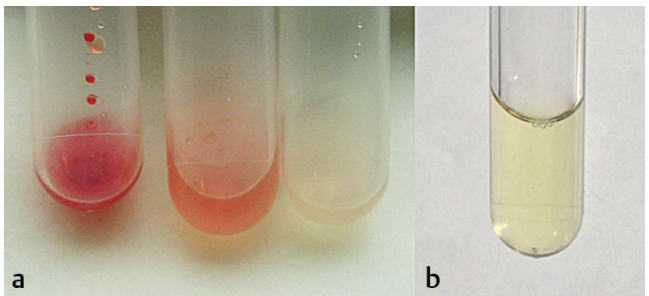

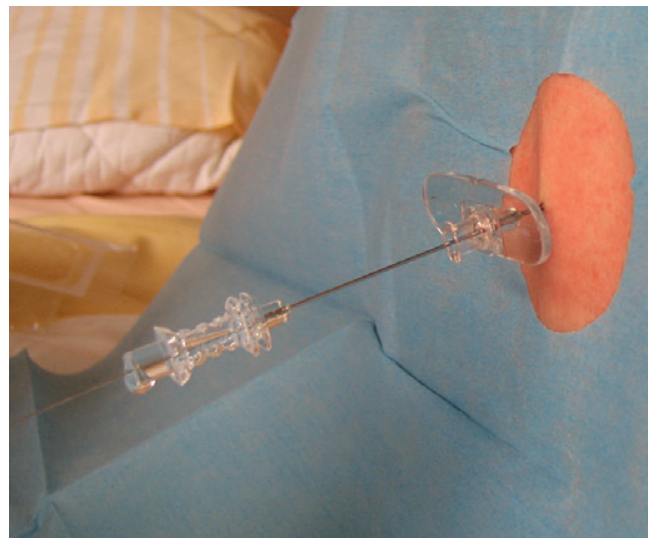

Abb. 5 Nach einem spürbaren Widerstandsverlust wird der Mandrin der Nadel zurückgezogen und geprüft, ob Liquor zurückfließt.

ner Besserung, ist ein epiduraler Blutpatch Mittel der Wahl. Hierbei werden ca. $10 \mathrm{ml}$ steril abgenommenes venöses Blut epidural appliziert. Dieses verschließt nach Koagulation das Liquorleck.

Früh bewegen I Nach der Lumbalpunktion wird eine Frühmobilisation empfohlen soweit es die Kreislaufverhältnisse zulassen. Eine postpunktionelle Bettruhe hat keinen protektiven Einfluss auf die Kopfschmerzentwicklung.

\section{Wichtiges in Kürze:}

- Vor Punktion sollten das Blutbild und der Gerinnungsstatus geprüft und der Patient über den Eingriff aufgeklärt werden.

- Achten Sie auf eine korrekte Lagerung.

- Atraumatische Sprotte-Nadeln sind zu bevorzugen.

- Die Schliffrichtung der Nadel bei Duradurchstich ist senkrecht.

- Führen Sie vor Entfernung der Nadel den Mandrin wieder komplett ein.

- Nach Punktion wird eine Frühmobilisation empfohlen.

- Bei postpunktionellen Kopfschmerzen Bettruhe und ggf. NSAIDs verordnen.

Interessenkonflikt

Die Autoren geben an, dass kein Interessenkonflikt besteht

\section{Weiterführende Literatur}

1 Deutsche Gesellschaft für Neurologie, Leitlinien für die diagnostische Liquorpunktion. Im Internet: http://www. dgn.org/leitlinien/11-leitlinien-der-dgn/2424-II84-2012-diagnostische-liquorpunktio; Stand: 18.03.2015

2 Straus SE, Thorpe KE, Holroyd-Leduc J. How do I perform a lumbar puncture and analyze the results to diagnose bacterial meningitis? JAMA 2006; 296: 2012-2022

3 Ellenby MS, Tegtmeyer K, Lai S, Braner DA. Videos in clinical medicine. Lumbar puncture. N Engl J Med 2006; 355: e12

4 AWMF online, Rückenmarknahe Regionalanästhesien und Thromboembolieprophylaxe/antithrombotische Medikation. Im Internet: http://www.awmf.org/ leitlinien/detail/II/001-005.html; Stand: 18.03.2015
DOI 10.1055/s-0041-102251 140: $738-740$

(c) Georg Thieme Verlag KG . Stuttgart · New York . ISSN 0012-0472 